\title{
An Energy Efficient Load Balancing Protocol for Multi-Hop Clustering in Wireless Sensor Network
}

\author{
M.Jeyakarthic, S.Manikandan
}

\begin{abstract}
Cluster based WSNs is a rising and empowering technical knowledge with the achievable to revolutionize Data Communication Technology. The purpose of WSN stretch out to diverse areas such as the security and surveillance, Medical and Health, Military related application, Agriculture, Entertainment and so on. In wireless sensor networks (WSNs), the sensor nodes are highly distributed in order to sense and transform information to base station. However, the major challenge in WSN is to avoid collision and energy dissipation due to redundant data and thereby extending the network lifetime. To address this issue, a novel energy efficient load balancing protocol (EELB) for data forwarding in multi-hop clustering based WSN is proposed. EELB is a hierarchal cluster-based protocol which schedules the sensor nodes to different modes namely sleep mode and active mode by probing the data transformed to decrease energy consumption effectively. A sensor node is set to sleep mode when it senses and transfers redundant data for an extended time. The other sensor nodes remain enabled in active mode for sensing and transmission of data packets. Also, the proposed protocol selects a reliable cluster head based on remaining residual energy level and trust value of each node. The Simulation outcomes depicts that the proposed EELB protocol performs well than conventional protocol with respect to average energy consumption, lifetime of nodes and the Packet Delivery Ratio.
\end{abstract} node

Keywords : Clustering, Load balancing, Scheduling, Sensor

\section{INTRODUCTION}

Cluster based WSNs is said to be a crew of spatially dispersed and dedicated sensor for observing and reporting the environmental states of the surrounding and congregate the information at a central location. Clustered WSNs are widely employed to measure the atmospheric conditions like temperature, sound, humidity, wind, pollution levels and so on. Recently, there has been advanced research carried out in wireless communication, which made it probable to develop wireless sensor networks (WSN) entailing of small devices [1]. These small devices are called sensor or nodes, which congregate information by sensing its environment. These sensing nodes comprises of CPU, memory, battery and transceiver for data processing, data storage and for transmission of data or signals. Since, sensor nodes in WSN

Revised Manuscript Received on December 30, 2019.

* Correspondence Author

Dr,M.Jeyakarthc*, Assistant Director (Academic), Tamil Virtual Academy, Anna University Campus, Chennai, India.

S.Manikandan, Assistant Professor, Department of Computer Applications, Government Thirumagal Mills College, Gudiyattam, Vellore, India.

(C) The Authors. Published by Blue Eyes Intelligence Engineering and Sciences Publication (BEIESP). This is an open access article under the CC BY-NC-ND license (http://creativecommons.org/licenses/by-nc-nd/4.0/) are capable of sensing its environment incessantly, in certain situation the nodes senses and transfer superfluous data continuously which may lead to collision and energy depletion which affects the network life time and gradually the built - in battery will die. Also, there might arise situation where it is impossible to substitute exhausted batteries due to its cost prohibitive nature and hostile environment [2] Henceforth, in this paper an efficient energy routing protocol with sleeping schedule mechanism and reliable cluster head election algorithm is proposed.

In recent years, in wireless sensor network many researches are carried out in an attempt to overcome energy efficiency problem and discovered energy efficient protocols, algorithm and network architecture considering all aspects. A considerable number of protocols to increase the energy efficiency in WSNs have been built in past. These protocols could be categorized into flat based protocols and hierarchical based protocols and it is found that hierarchical protocols are more reliable as its support cluster routing [4]. clustering is recommended to WSNs because of its advantage. In cluster based WSN, the sensors are seprated into small groups called "clusters". The cluster consists of a node called "cluster head $(\mathrm{CH})$ ", which is responsible for managing the entire cluster [5]. A number of small groups called "clusters" which manages the whole cluster. A sample cluster based wireless sensor network architecture is shown in below Fig. 1.

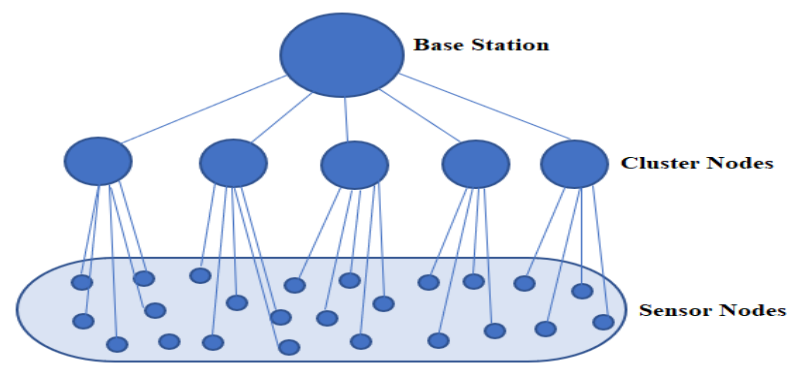

Fig.1 WSN Architecture

In WSN, the cluster head drops in energy level than the other nodes due to their frequent data transmission to the sink nodes. The problem of energy depletion at cluster head can be can be avoided by efficient load balancing technique and by electing cluster heads in rotation. However, most of the algorithm selects cluster head selection dependent on remaining energy which may not suit various application. Hence, there is a need for efficient cluster head selection technique which considers other factors such as reliability, transmission coverage, prolonged node lifetime, robustness, trust management and simplicity.

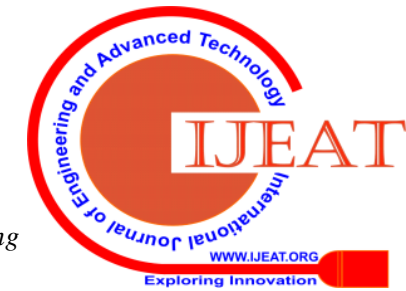


To resolve the above two stated problem, an energy-efficient load balancing (EELB) is proposed, were the cluster head is elected based on two parameters namely trust value of sensor and energy level of sensors. Also, EELB slate the sensor within the cluster into active or sleep mode to minimize the number of data transmitted to cluster head.

The remaining of this research paper is prearranged as follows: In section II "the work related with focus to energy aware routing protocols, cluster head selection and load balancing between the nodes is presented. We describe the details of our proposed protocol in "System model and Problem Statement" in section III. In section IV the proposed Energy efficient Load balancing routing protocol is briefly discussed. At Section V, the "Simulation Setup" is shown and their outcomes is discussed. To conclude this paper the "Conclusions and future work" is elaborated section VI.

\section{RELATED WORKS}

Wireless sensor network has emerged as a promising technology for enormous number of applications with different needs. however, there are yet few impediments to overcome before it becomes a mellowed technology. One of the vital impediments is the energy constraint suffered by sensor nodes. Recently, there are numerous researchers carried out to relating energy saving WSN. In this paper we are concern about energy conservation, load balancing scheme, cluster head selection schemes and their associated schemes. Heinzelman[6] proposed protocol known as "LEACH( Low Energy Adaptive Clustering System)". The key aspect of LEACH is that it separate nodes into group called clusters. A cluster head is selected for each group in order to communicate with the sink. For most part the cluster head is appointed primarily based on the highest level of energy remaining, so the cluster head can rule the node group for extended periods than the other nodes. However, this strategy of selection indicates only partial literary success. Mittal et al. [7] identified an enhanced LEACH protocol that has dual cluster nodes.in which a cluster head act as a primary cluster head and responsible for gathering of information from members of cluster and to transmit this data to Base station. The other cluster head is a Sub-Cluster head which act as a substitute for primary cluster head and kept for possible later use when the primary cluster head runs out of energy. This Sub-Cluster head is then accountable for all information transmission between the cluster and base station. Later, a variation of LEACH called "Hierarchical LEACH (H-LEACH)" [8] is designed which aims to decrease the level of depletion of energy by minimizing the distance data travelled. H-LEACH performs similar to LEACH in its initial cycles. H-leach differs from traditional leach by selecting a master cluster head intended for all cluster. The master cluster head is the key in charge for transmitting the data with optimal distance to base station.

$\mathrm{L} \mathrm{u}$ a $\mathrm{n}$ et al. [9] suggested a technique founded on LEACH by solidifying degree of nodes and residual energy of WSNs. The cluster head are chosen depending on sum of nodes connected and the maximum remaining energy. Younis et al. [10] developed a protocol called HEED which is a multi-hop clustering algorithm. In HEED, to elect a cluster head two of its parameters are utilized namely communication cost and remaining energy. The communication cost is termed as cost of communication between the members of cluster. However, the major limitation of HEED is, in certain situation cluster heads which are near to base station run out of energy due to hotspot issue. Ankit et al. [11] designed a chain-based routing protocol over LEACH called LEACH-CC. The LEACH-CC utilizes a centralized clustering algorithm in order to decrease energy consumption and deferral to expand the time of network lifetime. Although LEACH can build the network lifetime, yet at same time there still remain issues associated with assumptions used in this protocol.

Wu et al. [12] presents a dynamic scheduling mechanism and the experimental results proved to have improved network life time than the fixed sake-up mode of LEACH. In [13], to minimize the energy consumption of entire WSN an tree-based clustering routing protocol with sleep scheduling is proposed. This protocol preserve energy by shutting down radio frequencies of unnecessary nodes. More et al. [14] put forth a sleep protocol called "Random Back off sleep protocol" to extend the network period and to balance the depletion of energy among the sensors. RBSP make sure that the likelihood of neighbour nodes turning into lively node is contrarywise associated to the level of energy depletion in current active node. However, the limitation of RBSP is the time, cost and energy associated in building the models. Thus, from reviewing the literature there are following issues which are yet need to be resolved are stated below:

- Minimizing the energy depletion in multi-hop clustered WSN

- Electing a reliable cluster head

- Providing load balance within intra cluster environment and perform energy consumption

- Thus, Energy efficiency and load balance remain an open problem in WSN environment.

The contributions of the paper is summarized below.

- We propose an Energy Efficient Load Balancing Protocol for multi-hop clustering in WSN.

- A reliable cluster head is elected, which upsurges the lifetime of the network. Also, sensor nodes are scheduled to sleep and active mode to achieve load balancing

\section{SYSTEM DESIGN AND PROBLEM STATEMENT}

\section{A.System Design}

The System is designed with following consideration, all sensor nodes with cluster based WSNs are identical in hardware component, software and data computing configuration. Also, initially, all the sensor nodes have same level of energy with a constraint that energy cannot be refueled. However, after certain operation, nodes may be of unequal energy levels depending upon its transmission. It is also considered that all the sensor is capable of switching for sleep mode to active mode or vice-versa. Subsequently, after distribution of the sensor nodes, they are logically partitioned into group of nodes and each group has a cluster head node which is solely authoritative for aggregating data from nodes and transferring the same to base station. We consider the transmission takes place in hierarchical fashion. As shown in below Fig..2

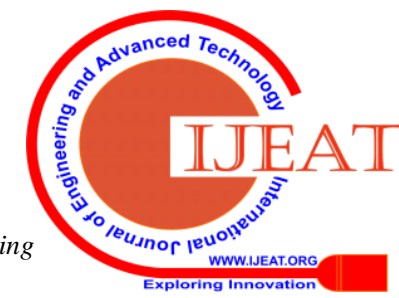




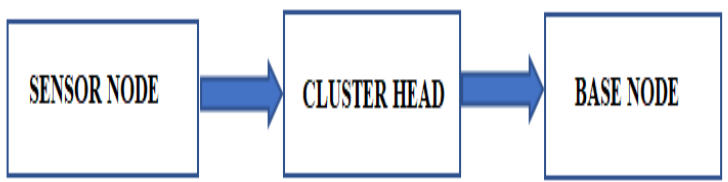

Fig.2 Cluster Head Election Algorithm

Again, transmission between cluster head and the base node take place in multi-hop fashion.

\section{B.Problem Statement}

The objective of the proposed problem is to design an efficient load balancing and energy conservation protocol for wireless sensor network. Since the sensor nodes are deployed with limited energy which cannot be refueled, the routing protocol should devour low energy and should not burden the nodes with continuous data transmission.

\section{PROPOSED ENERGY EFFICIENT LOAD BALANCING PROTOCOL(EELB)}

This section discusses the proposed EELB protocol elaborately. The proposed protocol is a hierarchal cluster-based protocol which uses multi-hop strategies to communicate with base station. The proposed EELB working is categorized into two distinct phases namely initial phase and active phase. In initial phase, the sensor nodes are positioned in respective environment. In order to provide energy efficient communication, the sensor nodes divide themselves into many clusters and a reliable cluster head is elected which is briefly discussed in 4.1(a) the active phase is solely responsible for data aggregation and conserving energy through sleep scheduling mechanism which is elaborately discusses in section 4.1(b)

\section{A. Initial Phase}

This phase starts once the sensor node are deployed in their respective fields. During this phase the sensor nodes self-organize themselves by dynamically dividing them into diverse clusters. For each cluster among the members a cluster head is elected during each round. However, selecting a reliable sensor node is still an open challenge. Therefore, in this paper the proposed EELB elects a reliable cluster using an efficient cluster election algorithm. The proposed algorithm elects a cluster head for each cycle considering remaining energy and trust value sensor node. The trust value of each node is determined by the number of successful transmissions of sending and receiving data. The trust value is determined by examining the routing table of sink node and each sensor node within WSNs. An outline of proposed election algorithm is shown in below Fig..3

Procedure cluster Head Election

Begin
Input: Number of Nodes $\mathrm{N}$ and Clusters

If Round=Initial

then

Calculate Renergy for node $\mathrm{N}$

Elect node $n$ with maximum Renergy in each cluster as new $\mathrm{CH}$

Else Calculate Renergy and Ntrust value for Node N

Elect $\mathrm{N}$ with maximum Renergy and Ntrust

All Node $\mathrm{N} \leftarrow$ Broadcast new $\mathrm{CH}()$

Repeat step 5 to step 8 for all rounds

End

\section{Fig.3 Cluster Head Election Algorithm}

During, initial round the cluster head is elected considering only the signal strength since the trust value cannot be determined during initial round. The process of selecting cluster head is continued for Nrounds. An TDMA schedule is applied to all members of the cluster group to send message or data to newly elected cluster head. Electing a reliable cluster is significantly important because the cluster is the only node which gathers data from all members of cluster and passes over the aggregated data to base node. The protocol enters into active phase after forming cluster and electing cluster head during initial phase

\section{B. Active Phase}

The Active phase is known as data transmission phase and it is initiated once clusters are formed and cluster head is elected. During this phase, the sensor within the cluster senses the physical environment and communicates the sensed data to the respective cluster heads. During data transmission the cluster head plays a significant role by aggregating all the collected data and transmit it to the base station either through direct transmission or multi-hop transmission.in direct transmission the cluster head directly transfers the data to base station. If the base station is far away from the cluster head then the cluster head transmits the compressed data to next nearest cluster head and the process continues until the data is transmitted to base station. A sample multi-hop transmission is shown in below Fig..4

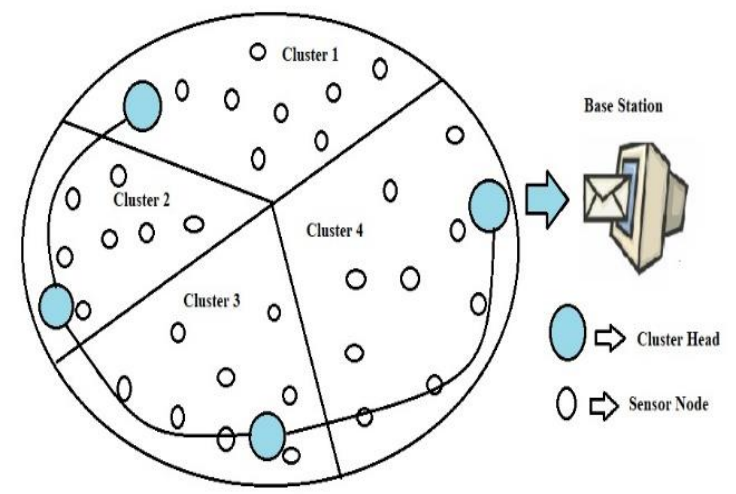

Fig. 4 Sample Multi-hop transmission between cluster heads 
However, at certain situation the sensor node transmits superfluous data for long time which causes energy depletion in sensor node. Hence to avoid depletion of energy the proposed protocol uses a sleep scheduling technique to balance the load in cluster head and sensors. The proposed sleep scheduling technique has two states namely active state and sleep state

Active State: In active state, the sensor node is allowed to sense the environment and transmit the data to cluster head

Sleep state: During sleep state the sensor node is temporarily aborted from sensing the environment. A sensor node is placed in sleep state when it sends superfluous data for a long time. The algorithm for sleep scheduling is shown below Fig. 3

Procedure Sleep Schedule

Begin

Input: Number of Nodes $\mathrm{N}$ and their Nodeid

For each node $\mathrm{N}$ in cluster monitor the data packets for time $\mathrm{T}$

If data packet transmits same data for Tinterval

then

Select Transmitting Nodeid

Set Nodeid $\leftarrow$ Sleep state ()

Else set other Nodeid $\leftarrow$ Active state ()

Broadcast sleepmsg () to Cluster Head

Repeat step 5 to step 8 for all rounds

End

\section{Fig.5 Sleep Scheduling}

Therefore, the proposed EELB protocol minimize the energy depletion and balance the load for cluster head and sensors. A new round is initiated after data transmission and the protocol implements initial and active state for each round. The performance of proposed EELB protocol in terms throughput, network life time, delay and efficiency are briefly discussed in following section

\section{SIMULATION OUTCOMES AND DISCUSSION}

The performance evaluation of proposed EELB is discussed in this section, the proposed protocol EELB is modelled using MATLAB. To assess the performance of proposed protocol the simulation outcomes are compared with other existing well-known protocols such as LEACH, H-LEACH, and HEED for following criteria's:

- Energy expended by each node with respect to the simulation time

- Number of nodes alive with respect to the simulation time

- Number of dead nodes with respect to the simulation time

- Packets transmitted to Sink with respect to Round

- Packets transmitted to Cluster Head with respect to Round

The Simulations parameter for experiments are following. The nodes are deployed randomly and clusters are formed based on EELB protocol in mixed network of a $100 \mathrm{~m} \times 100 \mathrm{~m}$ field size. The proposed EELB is a multi-hop protocol and follows multi-hop communication to base node. The sensors and the sink node deployed are immobile after the deployment and the sensor node battery is non rechargeable once it is deployed. Therefore, the energy of the sensor node is the important concern in our problem, like other routing protocols for WSN. in general, there two categories of energy: electronic energy and amplification energy but we consider only electronic energy. initial energy of sensor nodes is 0.5 Joules and size of a packet is 4000 bits. the widely-adopted parameters for simulation is shown in Table 1 .

Table.1 Simulation Parameters

\begin{tabular}{|c|c|}
\hline \multicolumn{2}{|c|}{ Simulation Parameters } \\
\hline Number of Nodes & 100 \\
\hline packet size & 512 \\
\hline Initial Energy & 0.5 Joules \\
\hline Network Area & $100 \mathrm{~m}^{*} 100 \mathrm{~m}$ \\
\hline Simulation Time & $500 \mathrm{Sec}$ \\
\hline Round Length & $2500 \mathrm{sec}$ \\
\hline Data Size & 500 bytes \\
\hline Broadcast packet size & 25 bytes \\
\hline
\end{tabular}

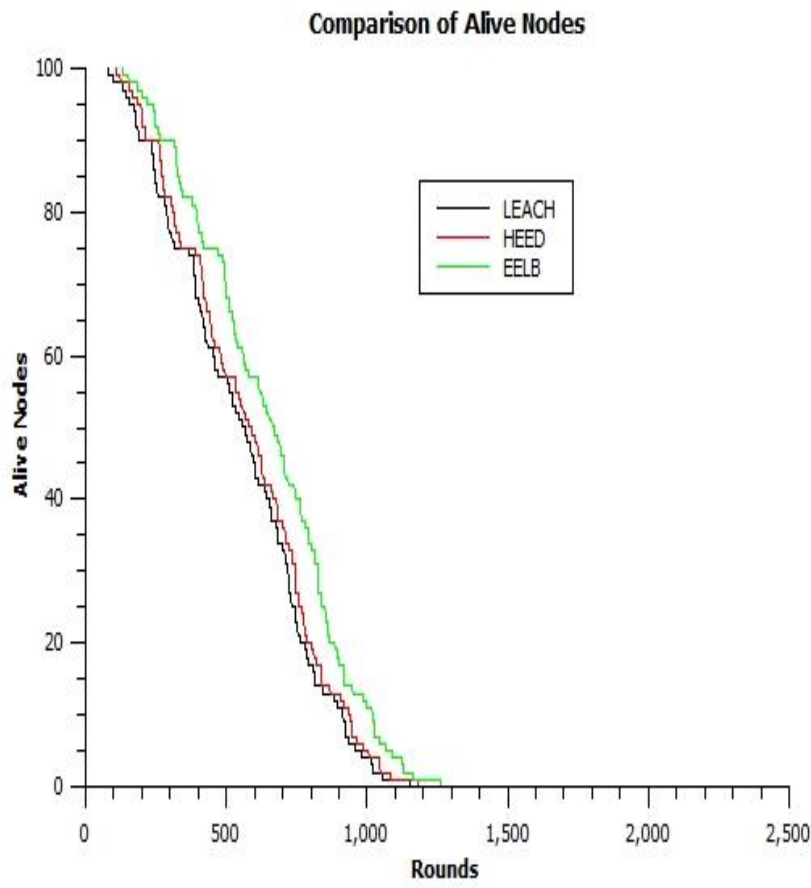

Fig.6 Comparison of Alive nodes

The above Fig.. 6 depicts the outcome of EELB in terms of number of alive nodes which indeed shows the network life time. The graph clearly displays that the number of node alive in EELB is higher existing HEED and the basic LEACH.Also, there is a difference of $10 \%$ in battery life. 


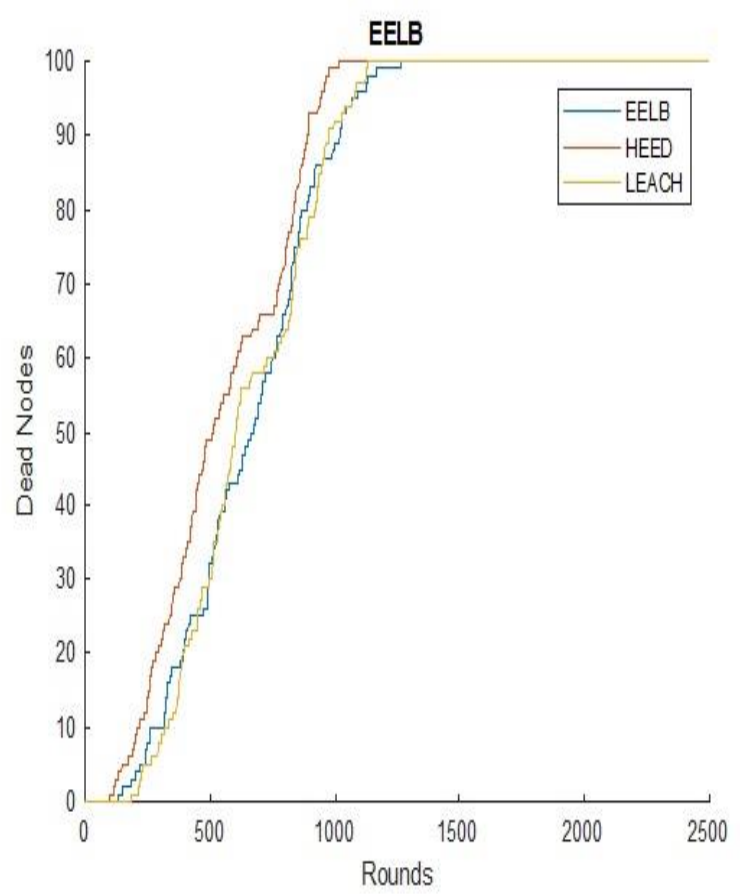

Fig.7 Comparison of Dead nodes

In Fig.7 the number of dead nodes with respect to round is shown. Due to effective load scheduling the energy available for proposed EELB increases thereby resulting in lesser dead nodes than the existing protocols.

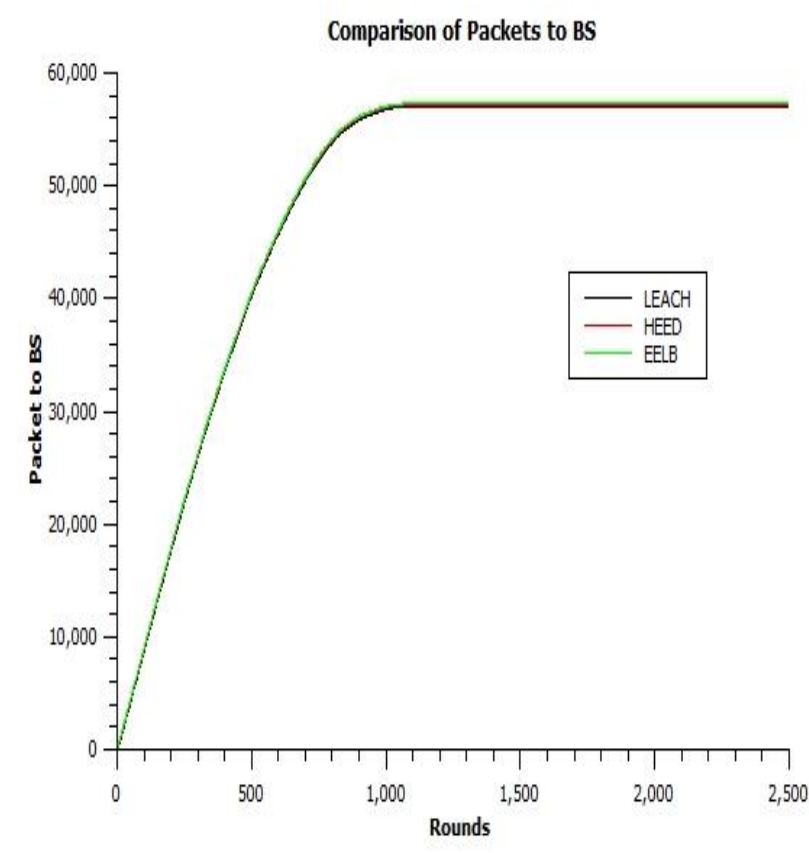

Fig. 8 Packets to Base Station

In above Fig..8 depicts the comparison of the packets transmitted to Base station and shows a slight variation of packets that are delivered to the Base station in EELB

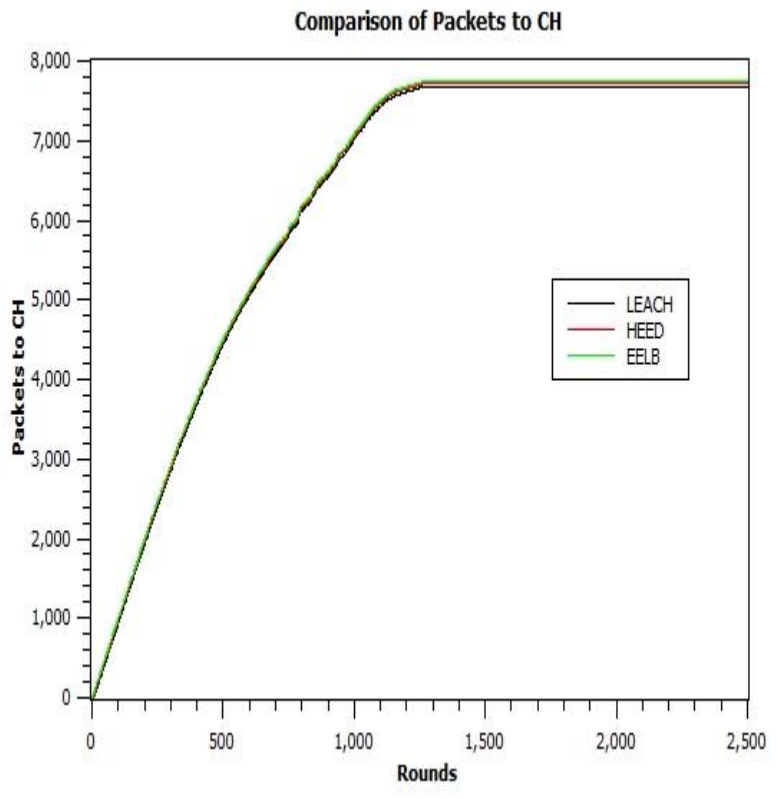

Fig.9 Packets to cluster Head

In Fig. 8 the comparison of the packets that received at the cluster head is displayed and the variation is visualized by 100 to 150 packets. Due to proposed load balancing technique the number of redundant transmissions has been reduced.

\section{CONCLUSION}

Cluster-based energy efficient load balancing (EELB) routing protocol is proposed in this paper with an objective to extend the lifetime of

network by balancing the load. The proposed EELB protocol employs the election algorithm to elect reliable cluster heads. In addition, the proposed protocol employs sleep scheduling algorithm to balance the load for both cluster head and sensor. The simulation outcomes are compared with standard existing protocols such as LEACH, H-LEACH and HEED. The results from simulation comparison proves that EELB protocol has best efficiency in terms of network lifetime and load balancing. Similarly, the EELB elects the most reliable cluster head for each round compared to existing protocols. Our future improvement is to enhance EELB by adopting dynamic traffic scenario and minimize the overhead due to control packets.

\section{REFERENCES}

1. Ayoub, Naeem, et al. "MAHEE: Multi-hop advance heterogeneity-aware energy efficient path planning algorithm for wireless sensor networks." 2017 IEEE Pacific Rim Conference on Communications, Computers and Signal Processing (PACRIM). IEEE, 2017.

2. Rault, Tifenn, Abdelmadjid Bouabdallah, and Yacine Challal. "Wsn lifetime optimization through controlled sink mobility and packet buffering." Global Information Infrastructure Symposium-GIIS 2013. IEEE, 2013.

3. Wan, Runze, and Naixue Xiong. "An energy-efficient sleep scheduling mechanism with similarity measure for wireless sensor networks." Human-centric Computing and Information Sciences 8.1 (2018): 18. 


\section{An Energy Efficient Load Balancing Protocol for Multi-Hop Clustering in Wireless Sensor Network}

4. Sarma, Hiren Kumar Deva, Avijit Kar, and Rajib Mall. "Energy efficient and reliable routing for mobile wireless sensor networks." 2010 6th IEEE International Conference on Distributed Computing in Sensor Systems Workshops (DCOSSW). IEEE, 2010.

5. Nurelmadina, Nahla, Ibtehal Nafea, and Muhammad Younas. "Evaluation of a channel assignment scheme in mobile network systems." Human-centric Computing and Information Sciences 6.1 (2016): 21.

6. Heinzelman, Wendi Rabiner, Anantha Chandrakasan, and Hari Balakrishnan. "Energy-efficient communication protocol for wireless microsensor networks." Proceedings of the 33rd annual Hawaii international conference on system sciences. IEEE, 2000.

7. Mittal, Nitin, et al. "Improved leach communication protocol for WSN." National Conference on Computational Instrumentation. Vol. 3. No. 1. 2010.

8. Wang, Wei, et al. "Leach-H: An improved routing protocol for collaborative sensing networks." 2009 International Conference on Wireless Communications \& Signal Processing. IEEE, 2009.

9. Luan, Weiping, et al. "An improved routing algorithm on LEACH by combining node degree and residual energy for WSNs." Internet of Things. Springer, Berlin, Heidelberg, 2012. 104-109.

10. Younis, Ossama, and Sonia Fahmy. "HEED: a hybrid, energy-efficient, distributed clustering approach for ad hoc sensor networks." IEEE Transactions on mobile computing 4 (2004): 366-379.

11. Gupta, Ankit, et al. "Clustering Approach for Enhancing Network Energy using LEACH Protocol in WSN." International Journal of Wired and Wireless Communications 2.1 (2012): 20-25.

12. Wu, Yan, Sonia Fahmy, and Ness B. Shroff. "Optimal sleep/wake scheduling for time-synchronized sensor networks with QoS guarantees." IEEE/ACM Transactions on Networking (TON) 17.5 (2009): 1508-1521.

13. Tan, Nguyen Duy, and Nguyen Dinh Viet. "SSTBC: Sleep scheduled and tree-based clustering routing protocol for energy-efficient in wireless sensor networks." The 2015 IEEE RIVF International Conference on Computing \& Communication Technologies-Research, Innovation, and Vision for Future (RIVF). IEEE, 2015.

14. More, Avinash, and Vijay Raisinghani. "Random backoff sleep protocol for energy efficient coverage in wireless sensor networks." Advanced Computing, Networking and Informatics-Volume 2. Springer, Cham, 2014. 123-131.

15. Wei Wang, Qianping Wang,Wei Luo,Mengmeng Sheng and Wanrong Wu,Li Hao "Leach-H: An Improved Routing Protocol for Collaborative Sensing Networks" international conference on Wireless Communications \& Signal Processing, 2009.

16. Rout, Rashmi Ranjan, and Soumya K. Ghosh. "Enhancement of lifetime using duty cycle and network coding in wireless sensor networks." IEEE Transactions on Wireless Communications 12.2 (2012): 656-667.Rout, R.R.; Ghosh, S.K. Enhancement of lifetime using duty cycle and network coding in wireless sensor networks. IEEE Trans. Wirel. Commun. 2013, 12, 656-667.

17. Lin, Kai, et al. "Balancing energy consumption with mobile agents in wireless sensor networks." Future Generation Computer Systems 28.2 (2012): 446-456.

18. Halder, Subir, Amrita Ghosal, and Sipra Das Bit. "A pre-determined node deployment strategy to prolong network lifetime in wireless sensor network." Computer Communications 34.11 (2011): 1294-1306.

\section{AUTHORS PROFILE}

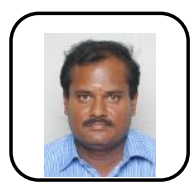

Dr. M.Jeyakarthic obtained his M.C.A. degree from Madurai Kamaraj University in 2000, M.Phil degree in Computer Science from Madurai Kamaraj University in 2005, M.B.A in E-Business from Annamalai University in 2008 and the Ph.D degree in Computer Science and Engg., from Annamalai University in 2016. He is an Assistant Director (Academic), Tamil Virtual Academy, Anna University Campus, Chennai, Tamilnadu, India. His specializations include Conten based image retrieval and data mining. His current research interests are Big Data analytics, Business intelligence, Wireless networks and pattern recognition.
Professor in Government Thirumagal Mills College, Gudiyattam, Velllore, Tamilnadu, India. His current research mainly on wireless sensor networks and data communication networks.
S.Manikandan obtained his Master of Science PostGraduate degree in Computer Science from Bharathidasan University on 2001, Master of Philoshopy degree in Computer Science from Periyar University in 2005, M.C.A lateral entry degree from Annamalai University in 2012 with distinction. He is an Assistnat

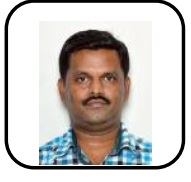

Retrieval Number: B3517129219/2019@BEIESP

DOI: 10.35940/ijeat.B3517.129219

Journal Website: www.ijeat.org
Published By:

Blue Eyes Intelligence Engineering

\& Sciences Publication 